\title{
Bienestar biopsicosocial en el plan de cuidados de enfermería al paciente adulto con VIH/SIDA
}

\author{
Alicia Carhuapoma-Tocto ${ }^{1}$
}

RESUMEN: El Síndrome de Inmunodeficiencia adquirida (SIDA) se asocia a una serie de infecciones y determinadas neoplasias en estadío avanzado de la enfermedad, afectando la calidad de vida de quien lo padece. El presente reporte de caso trata de un paciente adulto de 43 años de edad postrado, con antecedentes de tratamiento por SIDA y tuberculosis (TBC) que se encontraba internado en un hospital nacional de Lima Norte por complicaciones con el desarrollo de su enfermedad. Ante la referida problemática, en el presente reporte de caso se planteó realizar las intervenciones de enfermería, centrándose en la mejora de la calidad de vida del paciente enfocado en el bienestar biopsicosocial, para lo cual se desarrollaron cuatro diagnósticos reales como prioridad. El plan de cuidados se ejecutó durante dos semanas, empleando la valoración de los 13 dominios de la Taxonomía II de NANDA-I, según aspectos positivos y negativos y la Taxonomía y clasificación de los diagnósticos, intervenciones y resultados de enfermería: Interventions Classification (NIC) y Nursing Outcomes Classification (NOC) II. Mediante las intervenciones se consiguió mejorar los aspectos negativos priorizados como diagnósticos: "Desequilibrio nutricional: Ingesta inferior a las necesidades corporales", "Ansiedad", "Desesperanza", y "Baja autoestima situacional", de la misma manera se logró mejorar la calidad de vida del paciente con el fomento de su bienestar y satisfacción en este estadio de la enfermedad.

PALABRAS CLAVE: Atención de enfermería; Síndrome de Inmunodeficiencia Adquirida; Calidad de Vida; Ansiedad; Adaptación Psicológica.

Citar como: Carhuapoma A. Bienestar biopsicosocial en el plan de cuidados de enfermería al paciente adulto con VIH/SIDA. CASUS. 2017; 2(1):62-70. 


\section{INTRODUCCIÓN}

El programa conjunto de las Naciones Unidas sobre el Virus de la Inmunodeficiencia Humana VIH/SIDA (ONUSIDA) estimó que en el 20151.1 millones de personas en el mundo fallecieron por causas relacionadas a esta enfermedad. Así mismo una parte importante de muertes de las personas con VIH se debe a la tuberculosis (TBC), ya que de cada tres una se relaciona al contagio de la misma (1).

Estudios epidemiológicos internacionales y nacionales realizados en países de Latinoamérica, Europa, Asia y en el Perú coinciden que la calidad de vida de las personas que viven con VIH/SIDA es un tema de interés debido a que esta enfermedad va más allá del plano físico-biológico (2). Los aspectos económicos, socioculturales, psicológicos y ambientales inciden fuertemente en la calidad de vida de estas personas (2,3). El aspecto biológico también es afectado. Por ejemplo conservar un buen estado nutricional es fundamental para mantener un nivel adecuado de linfocitos CD4, lo cual contribuye a mejorar el sistema inmunológico y facilitar la acción de los antirretrovirales (4).

Ante la referida problemática, se planteó realizar las intervenciones de enfermería al paciente adulto en estadio SIDA, centrándose en fomentar el bienestar biopsicosocial del paciente a través de una atención humanizada y el empleo de intervenciones no farmacológicas. Las mismas serían complementarias a la administración de tratamientos tradicionalmente prescritos.

Lo anterior presenta implicaciones prácticas para la formulación, modificación o implementación de políticas públicas dirigidas a este grupo. Puesto que se orienta al abordaje de mejorar la calidad de vida del paciente con VIH/SIDA mediante aspectos que trascienden lo estipulado en las guías y programas de atención y cuidados en esta enfermedad (5). Para llevar a cabo la resolución del caso se empleó el modelo de los 13 dominios de la Taxonomía II de North American Nursing Diagnosis Association (NANDA) y la clasificación de los diagnósticos, intervenciones y resultados de enfermería: Nursing Interventions Classification (NIC) y Nursing Outcomes Classification (NOC), respectivamente (6-8).

\section{PRESENTACIÓN DEL CASO}

Se presenta el caso de un paciente adulto de 43 años de edad con VIH/SIDA y TBC. El paciente fue internado en un hospital nacional de Lima Norte con signos vitales $\mathrm{T}^{\circ} 36.5{ }^{\circ} \mathrm{C}, 90 / 50 \mathrm{mmHg}$ de presión arterial, 112 pulsaciones por minuto, 22 respiraciones por minuto y con un recuento de linfocitos CD4 igual a 101/ $\mu 1$. Asimismo se le diagnosticó: trastorno ansioso depresivo, síndrome ascítico edematoso, hipoalbuminemia, trastorno hidroelectrolítico y anemia. Posteriormente le fue diagnosticado Sarcoma de Kaposi diseminado lo cual complicó el estado de salud del paciente, empeorando su estado de ánimo y llevándolo al rechazo de todo tipo de procedimiento. De la misma manera mostró síntomas depresivos, desesperanza, descuido personal, dificultad para dormir, inapetencia, náuseas, irritabilidad al contar sus dolencias y aislamiento social.

\section{VALORACIÓN GENERAL}

La valoración se llevó a cabo determinando los aspectos positivos y negativos utilizando para ello los 13 dominios de la Taxonomía II de la NANDA-I, enfocándose para las actividades en los aspectos negativos a nivel biopsicosocial y hacia la búsqueda de medidas de afrontamiento en pro de la mejora de la calidad de vida del paciente (ver Tabla 1). 
Tabla 1. Análisis de los dominios según aspectos positivos y negativos

\begin{tabular}{|c|c|c|}
\hline Dominios & Positivos & Negativos \\
\hline Promoción de la Salud & - Cumple tratamiento prescrito. & $\begin{array}{l}\text { - Antecedente de SIDA y TBC. } \\
\text { - Descuido personal. }\end{array}$ \\
\hline Nutrición & $\begin{array}{l}\text { - Adecuado turgencia de piel. } \\
\text { - Temperatura axilar } 36.8^{\circ} \mathrm{C} \text {. } \\
\text { - Suplemento proteico. }\end{array}$ & $\begin{array}{l}\text { - Náuseas. } \\
\text { - Sed aumentada. } \\
\text { - Poca masa corporal. } \\
\text { - Apetito disminuido. } \\
\text { - Hemoglobina } 9 \mathrm{~g} / \mathrm{dL} \text {. } \\
\text { - Hematocrito } 30 .\end{array}$ \\
\hline Eliminación e Intercambio & - Murmullo vesicular normal & $\begin{array}{l}\text { - Ascitis. } \\
\text { - Deposición semilíquidas. } \\
\text { - Orina Hematúrica. }\end{array}$ \\
\hline Actividad y Reposo & $\begin{array}{l}\text { - Ventilación espontánea. } \\
\text { - Frecuencia Cardiaca } 110 \\
\text { por minuto. } \\
\text { - Presión arterial } 90 / 70 \mathrm{mmHg} \text {. }\end{array}$ & $\begin{array}{l}\text { - Insomnio. } \\
\text { - Postrado en cama. } \\
\text { - Usa pañal. } \\
\text { - Fuerza muscular disminuida. } \\
\text { - Frecuencia respiratoria } 24 \text { por } \\
\text { minuto. }\end{array}$ \\
\hline Percepción / Cognición & $\begin{array}{l}\text { - Consciente de su enfermedad. } \\
\text { - Comunicación verbal. }\end{array}$ & $\begin{array}{l}\text { - Ansioso - depresivo. } \\
\text { - Labilidad emocional (llanto). } \\
\text { - Desesperanza. }\end{array}$ \\
\hline Autopercepción & - Acepta puntos fuertes y débiles. & - Sentimiento de fracaso e ira. \\
\hline Rol / Relación & - Soltero / Profesional. & $\begin{array}{l}\text { - Dependiente físico. } \\
\text { - Aislamiento social. }\end{array}$ \\
\hline Sexualidad & - Sexo masculino. & $\begin{array}{l}\text { - Mantuvo conducta social } \\
\text { de riesgo. }\end{array}$ \\
\hline Afrontamiento / Tolerancia al estrés & - No se observa. & $\begin{array}{l}\text { - Sentimiento de soledad } \\
\text { - Ansiedad. }\end{array}$ \\
\hline Principios vitales & • Religión católica. & - No se observa. \\
\hline Seguridad y Protección & $\begin{array}{l}\text { - Uso de cama con barandas } \\
\text { - Aislamiento. }\end{array}$ & $\begin{array}{l}\text { - Adenopatías en cuello. } \\
\text { - Catéter periférico. } \\
\text { - Zona de presión en coxis. }\end{array}$ \\
\hline Confort & • Buena relación con entorno. & - Dolor de miembros. \\
\hline
\end{tabular}




\section{FASE DIAGNÓSTICA}

De acuerdo a la valoración realizada al paciente se plantearon y priorizaron cuatro diagnósticos de enfermería de la Taxonomía II de NANDA - I.

\section{REALES}

- (00002) Desequilibrio nutricional: inferior a las necesidades corporales $\mathrm{r} / \mathrm{c}$ incapacidad para absorber los nutrientes, y efecto secundario al tratamiento e/p hemoglobina de 9 g/dL y hematocrito 30. Dominio 2, Clase 1.

- (00146) Ansiedad r/c necesidades no satisfechas e/p insomnio y tensión facial. Dominio 9, Clase 2.

- (00124) Desesperanza r/c pérdida de la creencia en un poder espiritual e/p manifestación verbal de culpa y la falta de implicación en los cuidados. Dominio 6, Clase 1.

-(00120) Baja autoestima situacional r/c alteración de la imagen corporal e/p verbalización de desánimo por su peso. Dominio 6, Clase 2.

\section{FASE DE PLANIFICACIÓN}

La elaboración del plan de cuidados se estableció considerando los aspectos negativos de la valoración, los diagnósticos de enfermería, los objetivos planteados, la selección de intervenciones de enfermería y el logro de los objetivos. Asimismo se tomó en cuenta un horario de visita adecuado durante el tiempo de rotación en el servicio a fin de establecer contacto con el paciente quien estuvo dispuesto a colaborar. Además se le brindó consejería y escucha activa para lograr la confianza necesaria en beneficio de su bienestar biopsicosocial (ver tabla 2).

\section{FASE DE EJECUCIÓN}

El plan de cuidados de enfermería se ejecutó en dos semanas y estuvo destinado a mejorar tanto el nivel fisiológico como el emocional y espiritual. Enfocándose principalmente en la desesperanza, el estado de ánimo y la autoestima. Para documentar dichas actividades se utilizó la Clasificación de Intervenciones de Enfermería (NIC). 


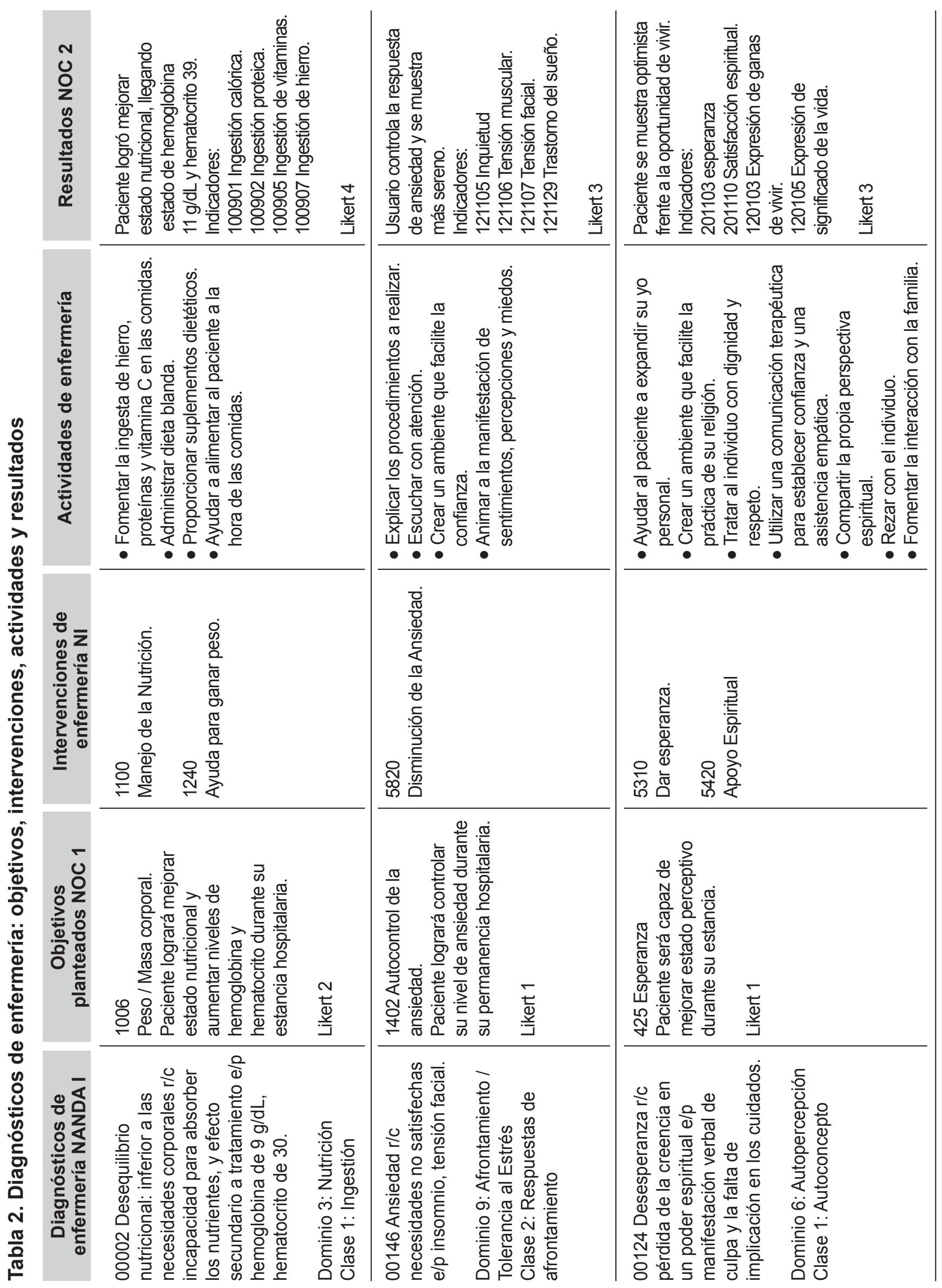



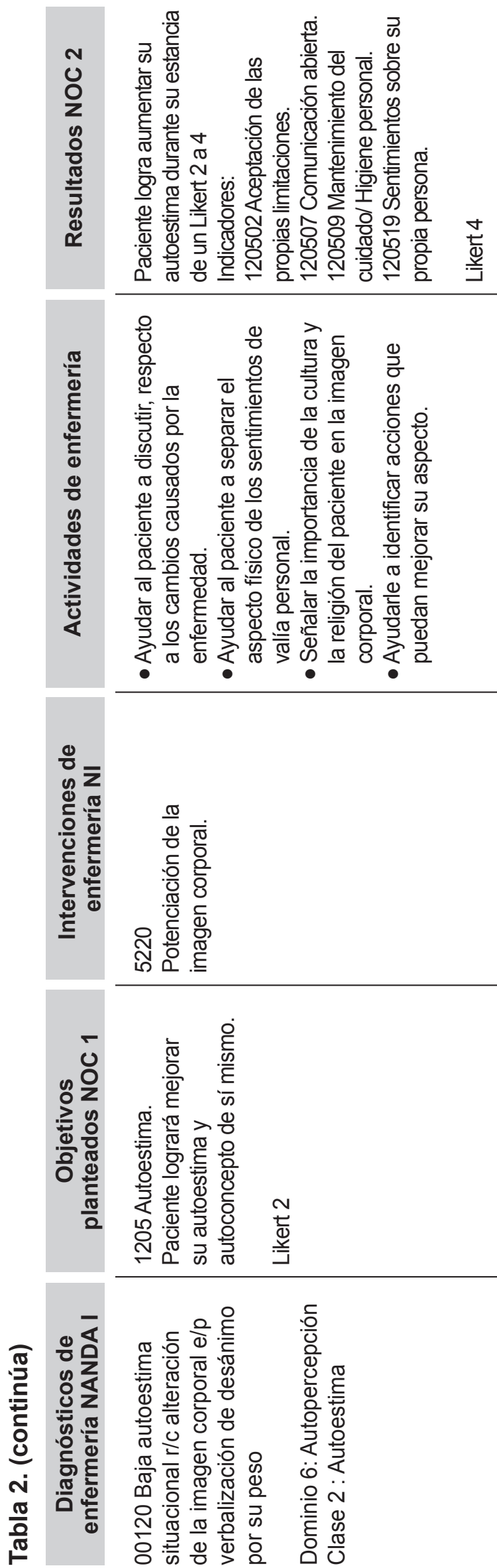


\section{FASE DE EVALUACIÓN}

Tal como se evidencia en la tabla 2 hubo una mejoría significativa y positiva en la resolución del caso. En el diagnóstico desequilibrio nutricional se consiguió incrementar de puntuación 2 a puntuación 4 en la escala de Likert, con la mejora de la alimentación por vía oral. Se le proporcionaron los nutrientes necesarios para cubrir las necesidades metabólicas y aumentar su hemoglobina a $11 \mathrm{~g} / \mathrm{dL}$.

El paciente fue diagnosticado con ansiedad, debida a la preocupación por las dolencias y al avance de la enfermedad, además de presentar insomnio puesto que descansaba solo de tres a cuatro horas con interrupciones. Por ello, mediante las actividades de enfermería se logró disminuir el nivel de ansiedad y favorecer el descanso, en un ambiente de seguridad, mejorando la puntuación de Likert de 1 a 3.

Respecto al diagnóstico desesperanza se consiguió mejorar el estado perceptivo vital del paciente, ya que refería que se sentía siempre igual, y persistían estos sentimientos y rechazaba todo tipo de procedimiento. Asimismo se logró mejorar el afrontamiento a través de las intervenciones de enfermería con una puntuación de Likert de 1 a 3 .

En cuanto a la autoestima, el paciente constantemente refería "me siento una nada", asimismo se encontraba hipoactivo, inapetente, insatisfecho con su imagen corporal y presentaba descuido en su higiene personal. Se logró potenciar la seguridad, confianza en sí mismo, interés por mejorar su aspecto físico y aceptar visitas. La escala de Likert fue de 2 a 4 .

\section{DISCUSIÓN}

En la evaluación del plan de cuidados se logró fomentar el bienestar biopsicosocial del paciente integrando las cuestiones biológicas, psicológicas y sociales hacia un mejor afrontamiento de esta condición. El paciente, en el aspecto biológico, logró mejorar el estado nutricional mediante la intervención manejo de la nutrición, aumentando su nivel de hemoglobina y hematocrito, reflejado en la escala de Likert de 2 a 4 . En ello, la comunidad científica reconoce que el cuidado nutricional puede contribuir a mantener la salud y a disminuir los efectos de una enfermedad crónica, en especial los afectados por el VIH (9).
Respecto a la ansiedad el paciente controló la respuesta nivel de ansiedad, logrando incrementar la escala de Likert de 1 a 3 disminuyendo su inquietud, tensión muscular, tensión facial y trastorno del sueño. Un estudio reciente confirma que con un entrenamiento a través de medidas no farmacológicas se puede lograr reducir la ansiedad, aumentar la capacidad de afrontamiento activo y la sensación de bienestar (10).

Frente al diagnóstico desesperanza, el paciente logró superar y fortalecer el aspecto de esperanza restableciendo un significado de la vida, mejorando la escala de Likert de 1 a 3 . Un estudio explica que la espiritualidad en el ser humano lo fortalece ante situaciones de cercanía a la muerte (11). Respecto a la baja autoestima situacional, el paciente logró aumentar su autoestima durante su estancia, ya que esta había sido afectada debido al estado avanzado de su enfermedad, a las complicaciones físicas y emocionales. Un estudio que consideró resultados similares encontró asociación estadísticamente significativa entre el nivel bajo de autoestima y el estadio avanzado de la enfermedad (12). El aumento de la autoestima favorece a las personas con VIH/SIDA en tener sentimientos positivos acerca de sí mismos. Por ello los enfermeros deben estar preparados para brindar una atención integral que considere tanto el aspecto biológico como los elementos psicológicos y espirituales teniendo en cuenta sus características emocionales y clínicas (13).

Lo anteriormente descrito, pone en evidencia la importancia de complementar los cuidados de enfermería, a través de medidas no farmacológicas, con las intervenciones que se llevan a cabo en base a una correcta valoración. Por ejemplo, en el aspecto nutricional, la enfermera tiene que proporcionar asesoramiento sobre requerimientos dietéticos relacionados con la terapia antirretroviral complementando la atención con un amplio equipo multidisciplinario en la consecución de los cuidados (14). Así mismo, otro estudio confirma que los enfermeros deben fomentar el bienestar biopsicosocial en estos pacientes, como factor de fortaleza y coadyuvante en el afrontamiento de su estado de salud (15). 
Entre las dificultades encontradas al poner en práctica el plan de cuidados fue la falta de privacidad debido a que el ambiente era compartido con otros pacientes. Por otro lado no hubo la continuidad deseada en las intervenciones no farmacológicas.

Se recomienda optimizar los cuidados de enfermería, fomentando el bienestar biopsicosocial con la finalidad de mejorar la calidad de vida de los pacientes afectados por el VIH/SIDA. Estos cuidados no se especifican en las guías y programas nacionales de atención de esta enfermedad. Se concluye que se alcanzó mejorar el bienestar biopsicosocial del paciente a través del fortalecimiento de medidas de afrontamiento relacionados al aspecto nutricional, ansiedad, desesperanza y autoestima personal.

\section{REFERENCIAS BIBLIOGRÁFICAS}

1. Programa Conjunto de Naciones Unidas sobre el VIH/ SIDA. Estadísticas mundiales 2016 [internet]. Ginebra, Suiza: ONUSIDA; 2016 [citado el 24 de febrero de 2017]. Disponible en : http://www.unaids.org/es/resources/f act-sheet

2. Cardona J, Higuita L. Impacto del VIH / SIDA sobre la Calidad de Vida: Metaanálisis 2002 - 2012. Rev Esp Salud Pública. 2014;88(1):87-101.

3. Tavera M. Calidad de Vida relacionada a la salud en pacientes con VIH. Revista Peruana de Epidemiología. 2010;14(3):170-176.

4. Cardona J, Higuita L. Calidad de vida en personas con VIH/SIDA pertenecientes a una organización no gubernamental. Medellín (Colombia), 2009. Salud Uninorte. 2011;27(2):236-246.

5. Cardona J, et al. Calidad de vida relacionada con la salud en adultos con VIH/ Sida, Medellín, Colombia, 2009. Biomédica.

2011;31(1):532-544.
6. Herdman TH, Kamitsuru S. NANDA International. Diagnósticos Enfermeros: Definiciones y Clasificación, 2015-2017. 10ma ed. Barcelona: Elsevier; 2015.

7. Bulechek GM, Butcher HK, Dochterman JM, Wagner CM. Clasificación de Intervenciones de Enfermería (NIC). 6ta ed. Barcelona: Elsevier; 2014.

8. Moorhead S, Johnson M, Maas ML, Swanson E. Clasificación de Resultados de Enfermería (NOC). 5ta ed. Barcelona: Elsevier; 2014.

9. Massip T, et al. Evaluación Nutricional de personas con VIH/Sida. Rev Chil Nutr. 2015;42(2):131-138.

10. Sánchez $S$, et al. Eficacia de un programa cognitivo - conductual para la disminución de la ansiedad en pacientes médicamente enfermos. Revista de Investigación Clínica. 2006; 58 (6): 540-546.

11. Montalvo A, Herrera A. SIDA: Sufrimiento de las personas con la enfermedad, Cartagena. Av Enferm. 2015;33(1):85-93.
12. Ayala F, Moreno J. Nivel de autoestima y su relación con el valor absoluto de linfocitos TCD4 en pacientes que viven con VIH/SIDA. Med Interna. 2015;31(3):146-153.

13. De Castro $C$, et al. Avaliação da autoestima em pessoas vivendo com HIV/AIDS no município de Ribeirão Preto - SP. Texto \& Contexto Enfermagem. 2013;22(4):1049-1055.

14. Gómez M. Intervención de la enfermera gestora de casos en el ingreso hospitalario de pacientes con infección VIH/SIDA [Tesis magíster]. Madrid: Universidad Complutense de Madrid; 2011.

15. Bastidas F. Abordaje espiritual en el acompañamiento a personas que viven con VIH/SIDA: Una perspectiva experiencial desde la fe cristiana. Revista Educación en Valores. 2009;1(11):99-115. 


\section{Biopsychosocial well-being in the nursing care plan for adult HIV/AIDS patient}

ABSTRACT: Acquired Immunodeficiency Syndrome (AIDS) is associated with a number of infections and certain neoplasms in an advanced stage of the disease, affecting the quality of life of those who suffer. The present case report is a 43 -year-old male patient with a history of treatment for AIDS and tuberculosis (TB) who is hospitalized in a national hospital in North Lima due to complications with the development of his disease. Given the aforementioned problem, in the present case report it was proposed to carry out the nursing interventions, focusing on improving the quality of life of the patient focused on biopsychosocial well-being, for which four real diagnoses were developed as a priority. The care plan was implemented for two weeks, using the 13 domains of NANDA-I Taxonomy II, according to positive and negative aspects and the Taxonomy and classification of nursing diagnoses, interventions and outcomes: Interventions Classification ) And Nursing Outcomes Classification (NOC) II. Interventions improved negative aspects prioritized as diagnoses: "Nutritional imbalance: Intake less than bodily needs", "Anxiety", "Hopelessness", and "Low situational self-esteem", in the same way it was possible to improve the quality of Life of the patient with the promotion of their well-being, and satisfaction at this stage of the disease.

\section{KEY WORDS:}

Nursing care; Acquired immunodeficiency syndrome;

Quality of life; Anxiety; Psychological Adaptation. 